\section{Roche gobbles Smarticles}

Roche has entered the arena of microbiology diagnostics with its August acquisition of Los Gatos, California-based GeneWEAVE. Roche will pay GeneWEAVE $\$ 190$ million upfront plus another $\$ 235$ million in product milestones for the biotech's Smarticles technology, a rapid phenotype-based system that detects live bacteria directly from clinical samples and assesses antibiotic susceptibility, a platform with potential uses in disease surveillance and testing in hospitals. GeneWEAVE, in stealth mode for several years, first publicly displayed its technology at the November 2014 meeting of the Association for Molecular Pathology. "There was a lot of expression of interest following that," says CEO Steve Tablak. Smarticles are based on non-replicating bacteriophages containing DNA probes that bind to a specific bacterial genus or family of bacteria combined with synthetically designed plasmids containing luciferase genes. On contact with the drug-resistant bacteria, the DNA construct is activated causing the bacteria to express luciferase, producing light, which can be detected in an assay. GeneWEAVE is competing with Tucson, Arizona-based Accelerate Diagnostics, which is developing a fluorescent in situ hybridization-based system, and a PCRbased system from BioFire, the molecular diagnostics affiliate of bioMérieux in Salt Lake City, Utah.

\section{Illumina opens Helix genomics store}

Gene sequencing giant Illumina has joined investors Warburg Pincus and Sutter Hill Ventures to raise in excess of $\$ 100$ million to form Helix, a consumer-focused genomics company based in the San Francisco Bay area. Helix will provide affordable exome sequencing and database services. The content will come from Helix's partners: applications developed on genomics-related topics such as medical education, nutrition and wellness, and genealogy. In this way, using an app store model, Helix will give entrepreneurs the ability to create new opportunities for consumers to use genomics without the burden of developing a laboratory and data security infrastructure. People will be able to make repeated inquiries of the data Helix secures and stores, which Helix hopes will stimulate the creative use of genomics information and lead to connections not yet envisioned. Helix will provide oversight to ensure that no inappropriate content is included, such as medical advice given without US Food and Drug Administration review. Helix has announced two initial partners: the Mayo Clinic and clinical testing giant LabCorp. the care that was taken in designing and implementing such a trial. The editors of The Lancet $(386,830,2015)$ in an accompanying editorial wrote, "That such a trial was even possible is a testament not only to the skill of the research teams but also to the commitment of communities to defeating an epidemic that has devastated their nation." According to Swati Gupta, Merck Vaccine's director of science and public health, the depth of the preclinical data was what enabled them to go directly into humans. "We were lucky. If we, for instance, were talking about another pathogen you wouldn't expect to start at the same place," she said.

The problem, however, is that with such a small number of Ebola cases, random occurrences cannot be completely ruled out. "If you have a relatively rare outcome-a couple of thousand people in each arm, but only a handful of Ebola cases-it allows the play of chance to impact your findings more. If by chance you had five cases among vaccine recipients that were deemed to be from exposure prevaccination, but in fact were vaccine failures, then the study findings change dramatically," says Lahey.

In a commentary published a few weeks later, Phillip Krause of the US Food and Drug Administration's (FDA's) Office of Vaccines Research and Review pointed out that according to a statistical test meant to reduce errors in an interim analysis, the trial did not meet statistical significance (Lancet 386, 831-833, 2015). He also explained that despite the study designers' attempts to rule out biases in cluster assignments, the delayed arm had more subjects per cluster than the immediate arm ( 57 versus 42 ), which increases the probability of detecting Ebola in the delayed arm. Michael Callahan, an infectious disease scientist at the Massachusetts General Hospital Vaccine and Immunotherapy Center, with more than a decade's experience treating Ebola in the Congo and Liberia, agrees with the FDA's cautionary tone. "The FDA is factually correct at this point, they haven't demonstrated $100 \%$ efficacy, and it's very unlikely that this vaccine will be $100 \%$ efficacious," he

\section{C "We're still in the grip of this silly belief if you pour more money into knowledge creation, you'll get more benefit. Let's have a conversation about the kinds of institutions we really need." Daniel Sarewitz, a professor of science and society at Arizona State University, commenting on a recent study showing that increased funding to biomedical sciences has not resulted in commensurate increases in health outcomes over the past five decades. (PNAS 112, 11335-11340, 2015)}

"Patients scooping companies on clinical trial data is a new frontier." Jason Napodano, an independent biotech analyst, commenting after two patients with spinal cord injuries receiving an experimental says. But what he is hearing from across US federal agencies is that the study designers should be "mindful of a forward thinking enrollment strategy in the region that will allow them to compare the current study with newly vaccinated subjects." Given these problems, the worry is that some may assume that studies of this kind are acceptable alternatives to randomly controlled trials in future outbreaks. But this is far from a fait accompli, says Lahey. "The problem with letting the phrase $100 \%$ efficacy out in the wild is that it makes it really difficult to have a reasoned and balanced conversation afterward. And that is exactly what we need to do, is have a reasoned conversation," he says. Jim Lavery, director of the Centre for Ethical, Social \& Cultural Risk at St. Michael's Hospital in Toronto, who is on the data safety monitoring board for a separate Ebola vaccine trial, agrees, but sees a silver lining. "One possible outcome of this whole Ebola story is that it may give us pause to revisit where we can find efficiencies in our testing processes without sacrificing the kinds of insights we need about efficacy and safety," he says.

Meanwhile, giving credence to the worry, a few more rings were added to the Ebola ça Suffit trial, even though the study group had stopped randomization, upon the advice of the data and safety monitoring board. "Presumably this was because with the drop in incidence, completing the trial as planned would not be realistic in a reasonable time frame and would put subjects at elevated risk despite considerable evidence of vaccine activity," says Paul Frankel, of the Division of Biostatistics at the City of Hope in Duarte, California. According to Gupta, the company is moving along two pathways. Long term, the goal is to get licensure from some regulatory group, and in the intermediate and short term, to get emergency use authorization in affected areas. "What they've managed to achieve is quite remarkable, and it's a very innovative design, and an extremely strong signal, no matter how you look at the data," she says.

Laura DeFrancesco Senior Editor

spinal scaffold developed by Bob Langer and commercialized by In Vivo Therapeutics, tweeted their progress causing stock fluctuations in the company. [The FDA regulates drug companies' social media postings, but has no such control over patients.] (The Boston Globe, 21 August 2015)

"This may be the clearest example where public opinion in China has likely played a role in stalling or stopping an innovation pathway that the government backs, for better or worse." Sam Geall, an anthropologist at the University of Sussex, speaking of GMOs, research into which has enjoyed government support, yet with a few exceptions, have not been allowed out of the laboratory. (The New Yorker, 31 August 2015) 